Precision Measurement and Fundamental Constants II, B. N. Taylor and W. D. Phillips, Eds., Natl. Bur. Stand. (U.S.), Spec. Publ. 617 (1984).

\title{
Spectroscopy of Stored lons
}

\author{
D. J. Wineland \\ Frequency and Time Standards Group, Time and Frequency Division \\ National Bureau of Standards, Boulder, CO 80303
}

\begin{abstract}
The benign environment and long confinement times obtained with ion storage techniques have led to some unique experiments in the area of precision measurements and fundamental constants. This is perhaps epitomized by the single electron $g$ factor measurements at the University of Washington in which a precision of 4 parts in $10^{11}$ has been attained. Now, use of lasers to cool stored ions has allowed the experimentalist to approach the goal of unperturbed atomic ions nearly at rest; most recently, spectroscopy has been periormed on single "cold" trapped ions. Stored ion experiments in the area of precision measurements and fundamental constants will be briefly reviewed. These include experiments on $\mathrm{e}^{-} / \mathrm{e}^{+} g$ factors, mass spectroscopy, lifetimes, and atomic spectroscopy. The intent is to emphasize the unique environment provided by ion storage techniques for these measurements.
\end{abstract}

Key words: atomic ion spectroscopy; electron $g$ factor; fundamental constants; ion storage; mass spectroscopy; precision measurements.

\section{Introduction}

The purpose of this paper is twofold. The first purpose is to outline "trapped ion" experiments of the last decade which have contributed to the area of precision measurements and fundamental constants. The second purpose is to emphasize why the stored ion "environment" is particularly well suited to the area of precision measurements.

The main advantage of the stored ion technique is that the ideal of an unperturbed species at rest in space is approached to a high degree. Specifically, charged particles such as electrons and atomic ions can be stored for long periods of time (essentially indefinitely) without the usual perturbations associated with confinement (for example the perturbations due to collisions with walls or buffer gasses in a traditional optical pumping experiment). These unique properties were exploited most notably by Dehmelt and co-workers [1] before 1970; since then, that group and others have continued to extend these techniques with dramatic results.

Unfortunately, there is a price to be paid for this property of long storage times with small perturbations-the number of particles that can be stored is typically small ( $\leqslant 10^{6}$ for a "trap" with centimeter dimensions); the resulting low densities are governed by the competition between space charge repulsion and the confining electromagnetic forces obtained under normal laboratory conditions. These low numbers, of course, require very sensitive detection techniques and preclude many types of experiments-for example spectroscopic experiments on complex molecular ions where only a small fraction of the ions is in a given state. Although some effort has been made to increase the number of ions by space charge neutralization [2, 3] and trap "arrays" [4], these methods introduce other experimental complications. In spite of the low numbers obtained, sensitive techniques have been developed to detect simple species such as electrons and atomic ions so that single electrons $[5,6]$ and ions $[7,8]$ have been observed.

Since the intent is to discuss experiments relating to precision measurements and fundamental constants, many interesting experiments using stored ion techniques are not discussed in this paper. The reader is referred elsewhere to experiments on, for example, photodetachment $[9,10]$, chemical reactions $[11]$, electron-ion recombination [12], charge transfer $[13,14]$, and nonneutral plasma studies [15]. Also, more general reviews are available $[1,16,17]$.

In Sec. 2, methods of trapping are briefly reviewed. In Secs. 3-6, experiments on electrons/positrons, mass spectroscopy, atomic spectroscopy, and lifetime measurements are described with emphasis on the ion's environment. In the last section, speculations about other future experiments are made.

\section{Ion Trapping Methods}

Four types of traps have been useful: the rf (or Paul) trap, the Penning trap, and electrostatic and magnetostatic traps. These traps and their relevant properties are briefly described here. More detailed descriptions are given elsewhere $[1,16]$.

\subsection{The of Quadrupole Trap}

The ideal rf or Paul [18] trap uses hyperbolic electrodes in a vacuum apparatus as shown in Fig. 1. These electrodes are symmetric about the $z$ axis, so we can describe the potential in cylindrical coordinates. If an alternating voltage of frequency $\Omega$ is applied between the endcaps and ring electrode, then the instantaneous potential inside the trap is given by (following the notation of Ref. [1]):

$$
\phi(r, z)=A\left(r^{2}-2 z^{2}\right), \quad A=A_{0} \cos \Omega t .
$$

An ion experiences an $\mathrm{rf}$ electric field such that its motion (the "micromotion") is $180^{\circ}$ out of phase with respect to the electric force. Because the electric field is inhomogeneous, the force averaged over one period ( $T=$ $2 \pi / \Omega$ ) of the micromotion is in a direction of weaker field amplitude (independent of the sign of the charge), i.e., 
(a) rf or Penning trap
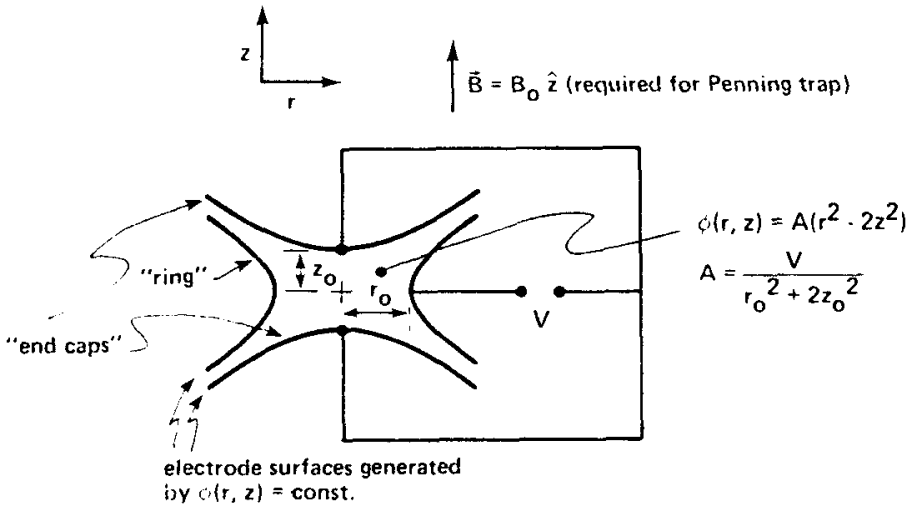

(b) Orbitron trap

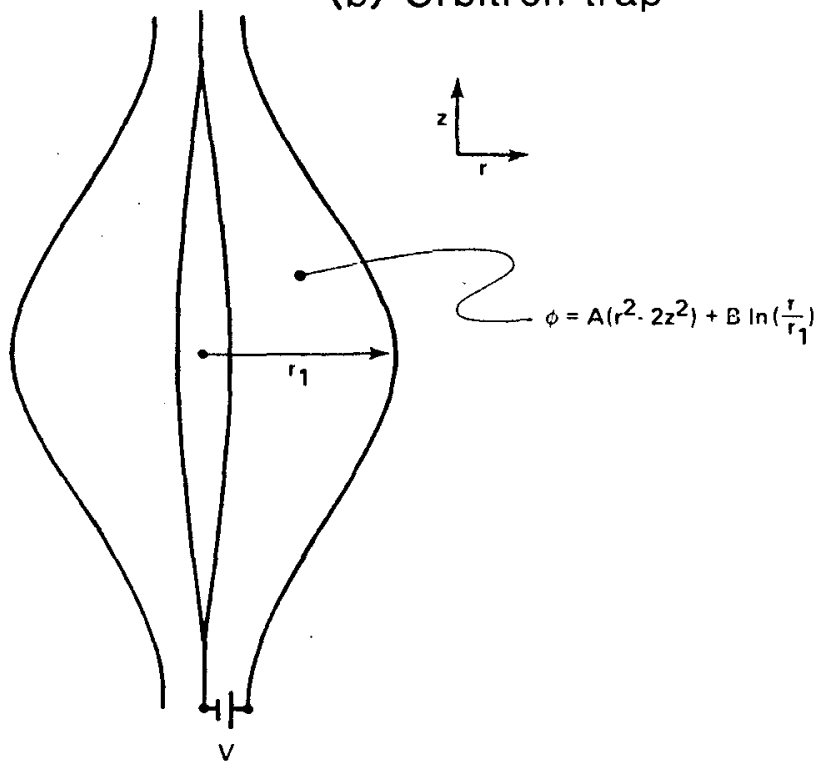

Figure 1. Electrode configuration for of, Penning, and orbitron ion traps. All electrode surfaces are figures of revolution about the axial (z) direction. In a practical orbitron trap, it is desirable to make the center electrode as thin as possible to reduce ion loss from collisions.

towards the center of the trap. For $\Omega$ sufficiently high, this restoring force gives rise to a pseudopotential

$$
\Psi(r, z)=\frac{e A \sigma^{2}}{M \Omega^{2}}\left[(\bar{r})^{2}+4(\bar{z})^{2}\right]
$$

where $\bar{r}$ and $\bar{z}$ are the positions of the ion averaged over $T, e$ is the ion charge, and $M$ is the ion mass. The resulting "secular" motion is

$$
\begin{aligned}
& \bar{z}=\bar{z}_{0} \cos \left(\bar{\omega}_{z} t+\phi_{z}\right) \\
& \bar{x}=\bar{x}_{0} \cos \left(\bar{\omega}_{r} t+\phi_{x}\right) \quad \bar{\omega}_{z}=2 \bar{\omega}_{r}=\frac{2 \sqrt{2 e A} A_{0}}{M \Omega} . \\
& \bar{y}=\bar{y}_{0} \cos \left(\bar{\omega}_{r} t+\phi_{y}\right)
\end{aligned}
$$

In more detail, we have for the $z$ motion:

$$
z=\ddot{z}_{0}\left[1+\frac{\sqrt{2 \omega_{z}}}{\Omega} \cos \Omega t\right] \cos \bar{\omega}_{z} t,
$$

where we require $\Omega / \bar{\omega}_{z} \gg 1$.

For ease of comparison with the Penning trap, it will be useful to consider the case of a spherical pseudopotential well in the $\mathrm{rf}$ trap. This is accomplished by simultaneously applying a static potential between the ring and endcaps so that

$$
\phi=\left(U_{0}+A_{0} \cos \Omega t\right)\left(r^{2}-2 z^{2}\right) .
$$

If we choose

$$
\begin{aligned}
U_{0} & =\frac{e}{M \Omega^{2}} A \sigma^{2}, \text { then } \\
\Psi(r, z) & =\frac{2 e A{ }^{2}}{M \Omega^{2}}\left[(\bar{r})^{2}+(\bar{z})^{2}\right] .
\end{aligned}
$$

In this case:

$$
\begin{gathered}
z=\bar{z}_{0}\left[1+\frac{2 \bar{\omega}_{z}}{\Omega} \cos \Omega t\right] \cos \bar{\omega}_{z} t \\
\left\{\begin{array}{c}
\bar{x} \\
\bar{y}
\end{array}\right\}=\left\{\begin{array}{l}
\bar{x}_{0} \\
\bar{y}_{0}
\end{array}\right\}\left[1+\frac{\bar{\omega}_{z}}{\Omega} \cos \Omega t\right] \cos \bar{\omega}_{r} t, \\
\bar{\omega}_{z}=\bar{\omega}_{r}=\frac{2 e A_{0}}{M \Omega} .
\end{gathered}
$$

where

Approximate experimental values for stored atomic ions are such that $r_{0}=1 \mathrm{~cm}, A_{0}$ is a few hundred volts $/ \mathrm{cm}^{2}$, $\Omega / 2 \pi$ is a few $\mathrm{MHz}$, and $\Omega / \bar{\omega}_{z} \approx 10$.

If the secular motion is "cold" then we expect the maximum density of ions to be given when the force from the space charge potential $\phi_{i}$ cancels that due to the trap. In this case, for the pseudo-potential given by Eq. (2), the ion cloud is a uniformly charged sphere of charge density $\rho$ with internal potential

$$
\phi_{i}=(2 / 3) \pi \rho\left(r^{2}+z^{2}\right) .
$$

Therefore for a well with $2 e A_{0}^{2} / M \Omega^{2}=10 \mathrm{~V} / \mathrm{cm}^{2}$, the condition $\phi_{i}=-\Psi(r, z)$ gives a density $n=3.3 \times$ $10^{7} / \mathrm{cm}^{3}$. In practice, attainable densities are approximately 1 to 2 orders of magnitude lower due to " $\mathrm{rf}$ heating" - a process which couples kinetic energy from the micromotion into the secular motion. One way this happens is through collisions with background gas. However, at high vacuum other coupling mechanisms can occur partly due to the presence of impurity ion s or imperfections in the trap electrodes. This process can give kinetic energies of ions of as much as electron volts and shortened storage times of less than $1 \mathrm{~s}$. If one uses a light buffer gas such as He to provide viscous damping [1] then storage times of many days can be achieved $[19,20]$. However, this increases perturbations due to collisions; therefore, for experiments on large numbers of ions in an rf trap, there appears to be a practical tradeoff between the temperature of the ions and possible perturbations due to collisions with a cooling buffer gas. However, this is not a fundamental limitation and one hopes it will be solved in the future.

We note that in principle, densities could be increased by increasing the rf field strengths; in practice, higher densities (close to $10^{9} / \mathrm{cm}^{3}[21]$ ) have been achieved by decreasing the electrode dimensions. This, of course, may not increase the ion number and it also may increase the rf heating because the field imperfections may be relatively larger.

It is interesting to note that the methods proposed for trapping of neutral particles in laser beams [22] are very analogous to the rf trapping of charged particles. In the laser beam case, the outer atomic electron experiences net forces in the (inhomogeneous) laser field. But since the electron is bound to the nucleus, dispersive effects 
occur. If the laser is tuned above the frequency of an electronic transition, similar to an ion in a $\mathrm{rf}$ trap, the atom is forced to regions of low field intensity. If the laser is tuned below resonance, the atom is forced to regions of high field intensity.

\subsection{The Penning Trap}

The Penning trap [23] uses the same electrode configuration as the rf trap (Fig. 1) but now $A$ in Eq. (1) is a constant $U_{0}$ such that the charged species see a static potential well along the $z$ axis. We have $\phi_{T}=$ $U_{0}\left(r^{2}-2 z^{2}\right)$. This causes a repulsive potential in the $x-y$ plane which can be overcome by superimposing a static magnetic field along $z\left(B=B_{0} \hat{z}\right)$. For a single ion in the trap, the equations of motion are $[1,24]$

$$
\begin{aligned}
\ddot{z} & +\omega_{z}^{2} z=0, \\
\omega_{z}^{2} & =\frac{4 e U_{0}}{M}=\frac{4 e V_{0}}{M\left(r_{0}^{2}+2 z^{2}\right)}, \\
\ddot{\mathbf{r}} & =\frac{1}{2} \omega_{z}^{2} \mathbf{r}-i \omega_{c} \dot{\mathbf{r}}, \\
\omega_{c} & =\frac{e B_{0}}{M c},
\end{aligned}
$$

where

$$
\mathbf{r}=x+i y
$$

Therefore

$$
z=z_{0} \cos \omega_{z} t \text { and } \mathbf{r}=\mathbf{r}_{c} e^{-i \omega_{c} t}+\mathbf{r}_{m} e^{-\omega_{m} m t}
$$

where

$$
\left\{\begin{array}{l}
\omega_{c}^{\prime} \\
\omega_{m}
\end{array}\right\}=\frac{1}{2} \omega_{c} \cdot\left\{\begin{array}{l}
+ \\
-
\end{array}\right\}\left[\left(\omega_{c} / 2\right)^{2}-\omega_{z}^{2} / 2\right]^{1 / 2} .
$$

Some useful expressions are:

$$
\begin{aligned}
& \omega^{2}+\omega_{z}^{2} / 2=\omega_{c} \omega \quad\left(\omega=\omega_{m} \text { or } \omega_{c}^{\prime}\right) \\
& \omega_{c}^{\prime}+\omega_{m}=\omega_{c} \\
& \omega_{c}^{\prime} \omega_{m}=\omega_{z}^{2} / 2 .
\end{aligned}
$$

Typical operating conditions in a Penning trap are such that for electrode spacings about $1 \mathrm{~cm}, V_{0}$ is a few volts and $B_{0}$ is larger than a few tenths of a tesla. The required large magnetic field can either be an advantage or disadvantage, depending on the experiment. It should be noted that the magnetron motion (the $\mathbf{r}_{m} e^{-i \omega_{, .,}}$term) is in an unstable equilibrium in the trap. For example, if collisions with background neutrals occur, the ions will diffuse out of the trap. (When the magnetron velocity is much less than the velocity of the neutrals, $r_{m}$ random walks in the $x-y$ plane with step size $\sim r_{c}$.) This is a potential disadvantage when compared to the rf trap for which all three degrees of freedom $(x, y, z)$ are in stable equilibrium. In practice however, this is not a limitation because ions can be stored for days in a room temperature apparatus [25] and electrons for weeks [5] in an $80 \mathrm{~K}$ apparatus. Moreover, the technique of sideband or radiation pressure cooling $[6,21,25,26]$ can reverse this diffusion process.

If the voltage $V_{0}$ applied to the electrodes becomes too high, then the radial electric field is high enough to overcome the ev $\times \mathrm{B} / \mathrm{c}$ magnetic force and the ions strike the ring electrode in exponentially increasing orbits (argument of square root in Eq. (5) becomes negative). For singly ionized atoms, the voltage where this occurs is given by $V_{\mathrm{c}} \approx 1200 B_{0}^{2}\left(r_{0}^{2}+2 z_{0}^{2}\right) / M$ where $V_{\mathrm{c}}$ is in volts, $B_{0}$ in tesla, $M$ in u (atomic mass units) and dimensions in $\mathrm{cm}$. This same mechanism limits the densities achievable in the Penning trap since space charge also gives radial electric fields. If the axial and cyclotron temperatures are low enough, then we can approximate the equilibrium distribution of the ion cloud [27] by a uniformly charged ellipsoid which has an (internal) potential of:

$$
\phi_{i}(r)=(2 / 3) \pi \rho\left(a r^{2}+b z^{2}\right)
$$

where $a$ and $b$ are constants. The $\mathbf{r}$ motion of an individual ion is now given by $\mathrm{Eq}$. (4) with

$$
\left\{\begin{array}{c}
\omega_{c}^{\prime} \\
\omega_{m}
\end{array}\right\}=\frac{\omega_{c}}{2}\{+\}\left[\left[\frac{\omega_{c}}{2}\right]^{2}-\frac{\omega_{z}^{2}}{2}-\frac{4 \pi e \rho a}{3 M}\right]^{1 / 2}
$$

where $\omega_{z}$ is the axial frequency for a single ion in the trap. For a spherical distribution, $a=b=1$; and the maximum density allowable (argument of square root term kept positive) is given by (using $\phi_{i}(z)=-\phi_{T}(z)$ ):

$$
n=\frac{p}{e}<\frac{B \sigma_{0}^{2}}{8 \pi c^{2} M}
$$

or

$$
n<2.7 \times 10^{9} B_{0}^{2} / M
$$

where $B_{0}$ is in tesla and $M$ in u. For $B_{0}=1 \mathrm{~T}, M=$ $100 \mathrm{u}, n<2.7 \times 10^{7} / \mathrm{cm}^{3}$ which is similar to the rf trap case. In experiments at the National Bureau of Standards (NBS), densities within a factor of about 3 of the theoretical maximum have been observed as indicated by the space charge shifted magnetron rotation frequency.

\subsection{Electrostatic Traps}

The orbitron trap [28] has the advantage of simplicity and although lifetimes are observed to be relatively short, precision lifetime and spectroscopic measurements have been made by Prior and his colleagues at Berkeley $[29,30]$.

The orbitron trap is purely electrostatic and has axial symmetry as shown in Fig. 1b. A potential which is useful is given by the general form [31]:

$$
\phi=A\left(r^{2}-2 z^{2}\right)+B \ln \left(r / r_{1}\right)
$$

because a harmonic well is provided along the $z$ axis. The equations of motion for the $x-y$ plane are not solvable analytically, but the motion is basically composed of precessing orbits about the $z$ axis [32]. Storage times in such a trap should be shorter because a single collision with a background neutral is sufficient to cause an ion to collide with the center electrode and be lost. Also the cooling discussed in Sec. 6 would be more difficult to realize. Nevertheless, with a trap whose electrodes approximate equipotentials of this function, trapping times of about one second were achieved by Knight [31] at a pressure of $10^{-6} \mathrm{~Pa}\left(\approx 10^{-8}\right.$ Torr). (The center electrode was a 100 $\mu \mathrm{m}$ dia. wire.)

An axial magnetic field superimposed along the $z$ axis has been investigated theoretically [33]; in this case, storage times should increase dramatically. Ions will eventually be lost by diffusing in towards the center electrode; therefore, storage times should be comparable to that of the Penning trap. However, the frequency of the drift motion about the center of the trap will depend on the distance from the $z$ axis; for the Penning trap this drift (magnetron) frequency is independent of radial posi- 
tion (neglecting space charge) and may be part of the reason for the slow evolution of the ion cloud under the influence of the ion-ion collisions. A possible advantage over the Penning trap is that experiments may be possible at much lower magnetic fields [33].

\subsection{Magnetostatic Traps}

Magnetic bottles are of course used extensively for plasma confinement and also in ion spectroscopy. The disadvantage in terms of spectroscopy appears to be that rather large magnetic field inhomogeneities are required for trapping, thus causing inhomogeneities and broadening in paramagnetic ion line shapes. The main application in terms of precision measurements has been the Michigan $\mathrm{e}^{+} / \mathrm{e}^{-}$experiments $[34]$; these are reviewed by $A$. Rich at this conference.

\section{3. $\mathrm{e}^{-} / \mathrm{e}^{+} \mathrm{g}-2$ Measurements in a Penning Trap}

This experiment has been pursued primarily by the groups of Dehmelt at the University of Washington, and Gräff at Mainz [35-37]. Only the Washington single electron experiment [6] will be discussed here, since its accuracy significantly exceeds that of the experiments on clouds of electrons [35-38]. Experiments on clouds of electrons are susceptible to rather large and difficult to measure space charge shifts. For example, the fractional shift of the cyclotron frequency of one electron due to another electron a distance $r$ away (in a direction perpendicular to the magnetic field) is approximately given by [39]

$$
\frac{\Delta \omega}{\omega_{0}}=\frac{1}{2} \frac{e}{m \omega_{c}^{2}}\left[\frac{1}{r} \frac{\partial}{\partial r}\left(r E_{r}\right)\right]
$$

where $m$ is the electron mass, $\omega_{c}$ is the cyclotron frequency, and $E_{r}$ is the electric field at one electron from the other. For a magnetic field of $1 \mathrm{~T}$, and $r=100 \mu \mathrm{m}$, $\Delta \omega / \omega_{0}=4.1 \times 10^{-9}$ which gives a shift of about $4 \mathrm{ppm}$ in $g-2$. In 1971, the Michigan group had measured the electron $g-2$ to $3 \mathrm{ppm} \mathrm{[40];} \mathrm{this} \mathrm{result} \mathrm{and} \mathrm{the} \mathrm{problems}$ associated with the space charge shifts led the Washington group to develop an experiment based on single electrons. A complete account of this experiment is given in Refs. [6, 41]. A simpler account is given in Ref. [42].

Briefly, a single electron can be detected $[5,42,43]$ by monitoring the currents induced in the electrodes by the axial $(z)$ oscillation at frequency $\omega_{z}$. The harmonically bound electron is equivalent to a series $\mathrm{L}-\mathrm{C}$ circuit which shunts the electrodes [5]; this L-C oscillator can be phase locked to an external oscillator by feeding back on the trap voltage $V_{0}[6]$. It is useful to think of the single electron bound to the Penning trap as a one electron pseudo atom whose "nucleus" is the earth (to which the Penning trap is fixed), thus the name "geonium" [6]. The quantum mechanical solution for the energy levels of this "atom" are given by:

$$
\begin{aligned}
E / h & =m v_{s}+(n+1 / 2) v_{c}^{\prime} \\
& +(k+1 / 2) v_{z}-(q+1 / 2) v_{m}
\end{aligned}
$$

where $m$ is the spin quantum number $(m= \pm 1 / 2)$ and $n$, $k$, and $q$ are the cyclotron, axial and magnetron quantum numbers $(n, k, q=0,1,2 \ldots)$. (The magnetron energy is mostly potential energy and is negative.)
In the $g-2$ and $e^{+} / e^{-}$mass ratio experiments it is necessary to detect the occurrence of spin flip and cyclotron transitions. This can be accomplished by superimposing a weak magnetic bottle centered on the trap such that the field is [6]:

$$
B=\left(B_{0}+\beta\left(z^{2}-r^{2} / 2\right) \hat{z}-\beta z y \hat{y}-\beta z x \hat{x} .\right.
$$

The spin, cyclotron, and magnetron magnetic moments interact with this magnetic bottle to shift the axial frequency by an amount

$$
\begin{gathered}
\delta v_{z}=\left[m+n+1 / 2+\left(v_{m} / \nu_{c}\right) q\right] \delta \\
\delta \simeq \mu_{B} \beta /\left(2 \pi^{2} m v_{z}\right)
\end{gathered}
$$

For $\nu_{z}=60 \mathrm{MHz}$ and $\beta=0.012 \mathrm{~T} / \mathrm{cm}^{2}, \delta \approx 1 \mathrm{~Hz}$, therefore for example, spin flip transitions would show up as 1 $\mathrm{Hz}$ changes in $\nu_{z}$ or as an accompanying step increase in feedback voltage to the locked axial resonance [6].

For reproducibility, it was desirable to have the electron at the center of the trap. This could be accomplished by sideband excitation $[6,26,44,45]$ as follows: with the (damped) axial motion driven by an inhomogeneous rf field of frequency $v_{z}+v_{m}$, the electron sees a sideband at $\nu_{z}$ because of the magnetron motion through the $\mathrm{rf}$ field. Therefore the electron absorbs quanta of energy $h\left(v_{z}+v_{m}\right)$ and reradiates (by the damping) quanta of energy $h v_{z}$. The deficit, $h v_{m}$, goes into the magnetron energy which shrinks the magnetron orbit. In principle, this technique allows for infinite storage times.

The electron $g-2$ has now been measured with an accuracy of $0.04 \mathrm{ppm}$ [46] and the positron $g-2$ to 0.05 ppm [47]. The details of these remarkable experiments are described in Ref. [6]; rather than give a complete description of the experiments, only the experimental environment is described here. From Ref. [6], the cyclotron degree of freedom was radiatively thermalized to $4 \mathrm{~K}$. The axial temperature was somewhat higher. Pressure was estimated to be as low as $10^{-12} \mathrm{~Pa}$ so that collisions with background gas could be neglected. At temperatures near $4 \mathrm{~K}$, axial excursions of $0.1 \mathrm{~mm}$ and cyclotron orbit sizes of $\sim 60 \mathrm{~nm}$ would be obtained. During cyclotron excitation to $n=15$, this gave a corresponding relativistic shift of only $-6 \times 10^{-9}$. Using sideband excitation, $r_{m}=14 \mu \mathrm{m}$. From Eq. (6b), there is, of course a large systematic shift $\left(\sim 6 \times 10^{-4}\right.$ in $\left.g-2\right)$ between the observed and free space cyclotron frequencies. Due to imperfections in the trap, a direct measurement of $v_{m}$ (by magnetron excitation) and the calculated value from $\mathrm{Eq}$. (6c) can disagree slightly. However, it can be shown that the error in $v_{m}$ is much less $[6,48]$ than the above difference which was measured to be $10^{-4} \nu_{m}$. Thus the error in $g-2$ was negligible. Errors in the present experiments are estimated from field jitter and drift and dependence of $g-2$ on applied spin flip power [47]. Ultimately, the experiment will be limited by systematic shifts associated with the superimposed magnetic bottle. To eliminate this problem, new ways have been proposed to detect the spin flips $[6,49]$. For example, it might be possible to use a switchable magnetic bottle so that while the cyclotron and $g-2$ resonances are driven, the bottle would be off and transitions would be detected by turning the bottle on [50]. Even without a magnetic bottle, the axial motion is still linked to the cyclotron degees of freedom via the relativistic mass shift $[6,51]$. This coupling is about an order of magnitude smaller than for the magnetic bottle; the required increase in axial well depth stability might be provided with a reference electron in an adjacent trap whose axial frequency is locked to a particular value [52]. 


\section{Mass Spectroscopy}

With the long storage times and high vacuums in the traps, one could expect extremely narrow motional resonances. In the Washington electron experiments cyclotron excitation resonances reproducible to a few parts in $10^{9}$ have been observed [53], and in principle the resolution should only be limited by effects due to inhomogeneous or time varying fields. Using the methods described in Sec. 3 , the electron/positron mass ratio has recently been measured to about $0.1 \mathrm{ppm}$ [63]. This very important experiment represents an improvement by more than two orders of magnitude over previous experiments. In addition, the cyclotron resonances of both atomic ions and electrons can be measured in the same magnetic field and in the same apparatus. This rather unique feature has led to experiments designed to make a direct measurement of the electron/proton mass ratio in a Penning trap.

The three current experiments using this method $[53-55]$ all basically measure the (shifted) cyclotron frequencies of electrons and protons in the same magnetic field. In the experiment of Gärtner and Klempt [54], resonances were detected by electron/proton loss; the largest uncertainty was due to a space charge shift in the proton cyclotron resonance. In the Van Dyck and Schwinberg experiment, resonances were detected in several (non-destructive) ways [53]; the limit here was the uncertainty in the relative positions of the electrons and protons in the trap and also possibly ion number (space charge) dependent shifts. In the experiment of Gräff, Kalinowsky, and Traut, resonances were detected by an energy dependent time of flight technique [55]. The experiment of Van Dyck and Schwinberg has the smallest uncertainty $(0.14 \mathrm{ppm})$ and substantial improvements can be expected.

With regard to space charge shifts, it is worth noting that in an ideal trap described by a quadratic electric potential and uniform magnetic field, if only one species is trapped and if the cyclotron motion is excited by a uniform electric field, then no space charge shifts can occur because only the center of mass mode is excited $[26,56]$. Unfortunately, deviations from the ideal trap fields can occur, impurity ions can be present, and the excitation usually occurs from non-uniform fields. All of these things can lead to (partial) excitation of the internal modes which then results in space charge shifts. These systematic shifts increase with the mass of the ion and therefore are much more important for protons and atomic ions than for electrons.

After the initial single electron work, it became apparent that it should be possible to observe single ion cyclotron resonances by observing the induced currents in the ring electrode if it is divided in half [57] or into quadrants [53]; the key advantage here is that space charge shifts are totally absent. Since the cyclotron frequency of protons in a field of $4 \mathrm{~T}$ is about $60 \mathrm{MHz}$, electronic detection should be similar to the detection of the axial resonance of single electrons.

Other, related mass spectroscopy experiments should be possible. First, using techniques as those referred to above, mass ratios of various atomic (molecular) ions could be measured. It should also be possible to observe the cyclotron resonance of single atomic ions by laser techniques. For example, if a single cold $\mathrm{Be}^{+}$ion is localized at the center of the trap [8] with a laser beam whose diameter is approximately $3 \mu \mathrm{m}$, then it should be possible to observe cyclotron excitation which increases the orbit radius to about $3 \mu \mathrm{m}$ by the decrease in fluorescence scattering. This corresponds to a temperature $\left(k_{B} T=(1 / 2) M\left(\omega_{c}^{\prime}\right)^{2} r_{c}^{2}\right)$ of $14 \mathrm{~K}$ in a field of $5 \mathrm{~T}$ and a relativistic shift of $1.4 \times 10^{-13}$. The axial and magnetron frequencies could be measured in a similar fashion. If the electron cyclotron frequency were measured in the same magnetic field, the accuracy in $M_{\mathrm{e}} / M_{\mathrm{p}}$ from this indirect experiment would be limited by the accuracy of $M_{\mathrm{Be}} / M_{\mathrm{p}}$ which is about $0.04 \mathrm{ppm}$ [58].

In preliminary experiments at NBS, the axial resonance $\left(v_{z} \simeq 200 \mathrm{kHz}\right)$ of a small cloud $(<10)$ of ${ }^{25} \mathrm{Mg}^{+}$ions has been measured in this way; however significant broadening (a few parts in $10^{4}$ ) is observed-presumably due to impurity ions. For an initial experiment on $\mathrm{Be}^{+}$, the electron cyclotron frequency can be measured indirectly by measuring the spin flip frequency on the same ion; since $g_{l}\left(\mathrm{Be}^{+}\right)$may be calculable to as good as a few parts in $10^{8}$ [59], the error in $M_{\mathrm{e}} / M_{\mathrm{p}}$ could still be limited by the error in $M_{\mathrm{Be}} / M_{\mathrm{p}}$. Ultimately when $M_{\mathrm{e}} / M_{\mathrm{p}}$ is known better by other means, such measurements should lead to better experimental determinations of $g_{J}\left(\mathrm{Be}^{+}\right)$ and $M_{\mathrm{Be}} / M_{\mathrm{p}}$.

We conclude by remarking that the extreme confinement possible with the stored ion techniques considerably relaxes the requirements on magnetic field homogeneity in these experiments.

\section{Radiative Lifetime Measurements}

Although perhaps not "high precision" in an absolute sense, the radiative lifetime measurements of Prior and colleagues at Berkeley should be included in this review because these measurements, made on simple atomic ions, can be compared with various theories. Lifetimes of ${ }^{1} S_{0} \mathrm{Li}^{+}(\tau \simeq 503 \mu \mathrm{s})$ in a Penning trap [60], 2s He${ }^{+}(\tau \simeq$ $1.92 \mathrm{~ms})$ in an orbitron [29], and $2^{3} \mathrm{~S}_{1} \mathrm{Li}^{+}(\tau=58.6 \mathrm{~s})$ in an rf trap [61] have been measured. One should also include the $5^{2} D_{3 / 2} \mathrm{Ba}^{+}(\tau \simeq 17.5 \mathrm{~s})$ measurement in an $\mathrm{rf}$ trap by Schneider and Werth [62] and the $5^{2} D_{52} \mathrm{Ba}^{+}(\tau \approx$ $47 \mathrm{~s}$ ) measurement in an rf trap by Plumelle et al. [19]. The key advantage of the traps in these measurements is the very long storage times and the benign environment which minimizes the effects of other relaxations. For the very long lifetimes, perhaps no other method is available.

\section{Atomic Spectroscopy}

As discussed in Sec. 1, the small number of ions involved makes it desirable to study simple atomic ions, but this does not preclude the study of simple molecular ions $[1,64]$. As we will see, the potential accuracy and precision for atomic spectroscopy using ion storage techniques is quite remarkable. This was apparent from the early experiments on the ${ }^{3} \mathrm{He}^{+}$ground state hyperfine structure $[1,65]$ where linewidths of about $10 \mathrm{~Hz}$ at 8.7 $\mathrm{GHz}$ were obtained. Since that time the $2 \mathrm{~s}{ }^{3} \mathrm{He}^{+}$hyperfine structure (hfs) has been measured in an orbitron trap by Prior and Wang [66]. The importance of this measurement is that a comparison between the $1 \mathrm{~s}$ and $2 \mathrm{~s}$ hfs suppresses the effects of nuclear structure corrections and allows an accurate check of the QED corrections; such experimental/theoretical comparisons are most meaningful for hydrogenic ions.

A significant interest in stored atomic ion spectroscopy stems from the desire to make a frequency standard. Stimulated by the $\mathrm{He}^{+}$experiments, various proposals $[65,67-78]$ have been made for microwave and optical stored ion frequency standards. For a microwave stand- 
ard, considerable attention has been given to the ${ }^{199} \mathrm{Hg}^{+}$ ion because (1) its ground state hyperfine frequency $(\sim 40$ $\mathrm{GHz}$ ) may be the largest of any conveniently usable ion, (2) its large mass gives a relatively small second order Doppler shift at a given temperature $\left(\sim 2 \times 10^{-13}\right.$ at 300 $\mathrm{K}$ ), and (3) a ${ }^{202} \mathrm{Hg}^{+}$lamp source $(194 \mathrm{~nm})$ is available for optical pumping. Starting with the work of Major and Werth [69], groups at Mainz [74], Orsay [76], and at least one commercial company [74] have sought to develop a frequency standard based on ${ }^{199} \mathrm{Hg}^{+}$ions stored in an $\mathrm{rf}$ trap. This work has been developed to a fairly high level; the group at Orsay [76] has made a working standard whose stability compares favorably with that of a commercial cesium beam frequency standard.

However, the full potential of the stored ion technique has yet to be realized. Historically, it appears that this has been due to two problems. (1) Because it has been difficult to cool the ions, second order Doppler or time dilation shifts can be sizeable (e.g.: $\sim 10^{-11}$ in Ref. [74], $\sim 4$ $\times 10^{-10}$ in Ref. [65]). (2) Signal-to-noise ratio has been poor due to the small number of stored ions.

In the past two or three years, both of these problems have been addressed. In 1978, groups at NBS and Heidelberg demonstrated $[21,25]$ that radiation pressure from lasers $[44,70,79]$ could be used to cool ions to temperatures $<0.1 \mathrm{~K}$, thereby reducing the second-order Doppler shift by more than three orders of magnitude below the room temperature case. As discussed below, the reduction of kinetic energy is most favorable for very small numbers of ions (down to one ion), so that there is a trade-off between the maximum number of jons we can use and the minimum second-order Doppler shift that can be achieved.

With regard to signal-to-noise ratio, in certain opticalpumping, double-resonance experiments, it is possible to scatter many optical photons from each ion for each microwave or optical "clock" photon absorbed [70, $73,79-81]$. This can allow one to make up for losses in detection efficiency due to small solid angle, small quantum efficiency in the photon detector, etc., so that the transition probability for each ion can be measured with unity detection efficiency. This means that the signal-tonoise ratio need be limited only by the statistical fluctuations in the number of ions that have made the transition $[78,81]$. Therefore the signal-to-noise ratio can be maximized for the given number of ions. The ability to sensitively detect the fluorescence from ions is emphasized by experiments on single ions $[7,8]$ where it is even possible to make photographs as shown in Fig. 2.

More recently, the narrow linewidths anticipated for the stored ion technique have been observed; a resonance linewidth of about $0.012 \mathrm{~Hz}$ at $292 \mathrm{MHz}$ has been observed [82] for the $\left(m_{l}, m_{J}\right)=(-3 / 2,1 / 2) \longleftrightarrow(-1 / 2,1 / 2)$ hyperfine transition of ${ }^{25} \mathrm{Mg}^{+}$at a magnetic field of about $1.24 \mathrm{~T}$ where the first derivative of the transition frequency with respect to magnetic field is zero. (The Ramsey interference method was implemented by applying two rf pulses of $1 \mathrm{~s}$ duration separated by $41 \mathrm{~s}$.) These narrow linewidths should be preserved with hyperfine transitions of higher frequency, such as in $\mathrm{Hg}^{+}$, but, of course, more attention must be paid to field homogeneity and stability. In any case, the above results allow one to contemplate microwave frequency standards with $Q>$ $10^{12}$ and inaccuracies $<10^{-15}[78]$. Of course, in addition to the interest in frequency standards, very high precision hyperfine and optical spectroscopy on $\mathrm{Hg}^{+}[74], \mathrm{Ba}^{+}$ $[20,82], \mathrm{Mg}^{+}[25,82,83]$, and other alkali-like ions [75] is possible.
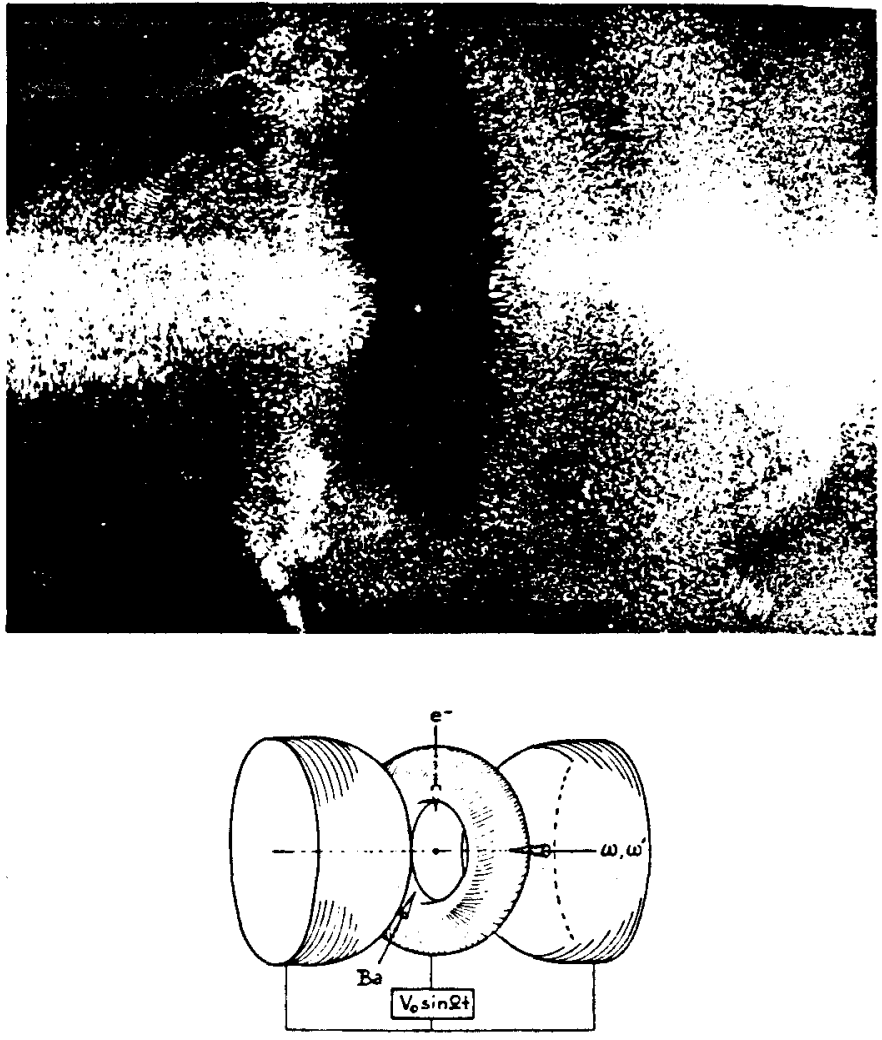

Figure 2. Photograph of fluorescence from single $\mathrm{Ba}^{+}$ion in an $r$ trap $(\mathrm{T}=10-86 \mathrm{mK}$; from Ref. [86]). The schematic diagram of the rf trap from same view. Inner diameter of ring is $0.7 \mathrm{~mm}$.

The possibilities for an optical frequency standard are even more dramatic $[70,71,77,78,84,85]$. For a given interaction time, the $Q$ of a transition will scale with the frequency. Therefore, in principle, an optical frequency standard would have clear advantages over a microwave frequency standard. The increased $Q$ would allow one to work with smaller numbers, even down to one ion $[70,77,78]$, and therefore obtain the "ultimate" atomic frequency standard. Example optical frequency standard candidates are the ${ }^{3} \mathrm{P}_{0} \leftarrow{ }^{1} \mathrm{~S}_{0}$ transition in $\mathrm{Tl}^{+}, \mathrm{Al}^{+}$or $\mathrm{Ga}^{+}$ [70], the ${ }^{3} \mathrm{P} \leftarrow{ }^{1} S$ transition in $B^{+}[85]$, the $D \leftarrow P \leftarrow \mathrm{S}$ Raman transitions in $\mathrm{Ba}^{+}$[77], the $\mathrm{S} \rightarrow \mathrm{D}$ two photon transition in $\mathrm{Hg}^{+}[71,78]$, and the ${ }^{2} \mathrm{P}_{3 / 2} \leftarrow{ }^{2} \mathrm{P}_{1 / 2}$ transition in $\mathrm{Pb}^{+}[72]$. All have $Q \geq 10^{14}$. Unfortunately, before the full potential of an ion-storage optical frequency standard can be realized, tunable lasers with suitable spectral purity must become available. This problem may be nearing solution [84]. Also, if an optical frequency standard is to provide time, the phase of the radiation must be measured. This appears to present a much more formidable problem $[84,85]$. Therefore it is expedient to continue to pursue a microwave frequency standard where both of these problems have been solved.

Rather than concentrate on the details of the various experiments it is useful to examine the environment of the stored ions in order to identify possible systematic effects. We will be primarily concerned with Stark and second order Doppler shifts. Usually only second order Stark shifts will be important; therefore we will be interested only in $\left\langle E^{2}\right\rangle$. As an example, for the strongly polarizable cesium (neutral) atom, the ground state hyperfine shift [88] is equal to: $\delta v / v=-2.5 \times 10^{-16} E^{2}(E$ in volts $/ \mathrm{cm}$ ); therefore electric fields of volts $/ \mathrm{cm}$ become 
important in rery high accuracy experiments [90]. Magnetic field shifts due to instabilities and inhomogeneities are of course important in the Penning trap but can be made negligible in many experiments. (See for example Refs. $[78,82]$.) Other effects such as electric quadrupole frequency shifts could be important in certain experiments $[70,78]$.

For single ions, laser cooling has already achieved temperatures between $10 \mathrm{mK}$ and $100 \mathrm{mK} \mathrm{[7,8].} \mathrm{Theoreti-}$ cally, when the motional oscillation frequencies $\Omega_{i}\left(\bar{\omega}_{z}\right.$ and $\bar{\omega}_{r}$ for the rf trap and $\omega_{z}$, $\omega_{c}^{\prime}$, and $\omega_{m}$ for the Penning trap) are less than the natural linewidth $(\Delta \nu=\gamma / 2 \pi)$ of the optical cooling transition, then the limiting "temperature" (governed by photon recoil) in each degree of freedom is given by $k_{B} T \cong \hbar \gamma / 2[21,25,44,87]$. (For a single ion, the precise minimum temperature depends on the angle of incidence of the laser beam and on the spatial distribution of recoil photons [87].) For strongly allowed transitions as in $\mathrm{Ba}^{+}$or $\mathrm{Mg}^{+}$, this limiting temperature is about $1 \mathrm{mK}$. For more weakly allowed transitions the temperature is correspondingly less but other limits such as thermodynamic limits can come into play [44]. When the condition $\Omega_{i} \gg \gamma$ is fulfilled then the limiting energy $[44,86]$ is given by $E_{i}=\hbar \Omega_{i}$ $\left(\left\langle n_{i}\right\rangle+1 / 2\right)$ where $\left\langle n_{i}\right\rangle=5 \gamma^{2} /\left(16 \Omega_{i}^{2}\right)$. Therefore the limiting kinetic energy is given by $E_{k i}=\hbar \Omega_{i} / 4$ but the uncertainty in energy (and therefore second order Doppler effect) is much less [44]. For simplicity we will assume only the case $\Omega_{i} \ll y$ below, however even better results are potentially obtained for the opposite condition. For a single ion in an rf trap, when $U_{0}=0$ the nonthermal micromotion has an average kinetic energy equal to that of the secular motion [1]; this is also true in the spherical trap. In the Penning trap the kinetic energy in the nonthermal magnetron motion can be much less than in the cyclotron or axial modes. Therefore, the minimum second order Doppler shifts are given approximately by:

$$
\frac{\Delta \nu_{D}}{v_{0}}=\frac{E_{k}}{M c^{2}}= \begin{cases}\frac{3}{2} \hbar \gamma / M c^{2} & \text { rf trap } \\ \frac{3}{4} \hbar \gamma / M c^{2} & \text { Penning trap }\end{cases}
$$

For a single ion in an rf trap (assuming the spherical trap of Eqs. (2) and (3)), $\left\langle E^{2}\right\rangle$ is primarily due to the oscillating $\mathrm{rf}$ fields and is largest for the $z$ motion. A simple calculation gives $\left\langle E^{2}\right\rangle_{z}=M \Omega^{2} \hbar \gamma / e^{2}$ for maximum laser cooling or $\left.\angle E^{2}\right\rangle_{z}=2 M \Omega^{2} k_{B} T / e^{2}$ for a given temperature in the $z$ secular motion. For a single ion in a Penning trap, it is usually possible to make $r_{m}, r_{c} \ll z$ $[8,87]$, therefore Stark shifts from the static fields are primarily due to the $z$ motion. We find $\left\langle E^{2}\right\rangle_{2}=$ $\hbar \gamma M \omega_{z}^{2} /\left(2 e^{2}\right)$ for maximum laser cooling or $\left\langle E^{2}\right\rangle_{z}=$ $k_{B} T M \omega_{z}^{2} / e^{2}$ at temperature $T$. In the Penning trap, a larger effect can be caused by the motional electric field $\mathrm{E}=\mathrm{v} \times \mathrm{B} / c$. We have $\left\langle E^{2}\right\rangle_{M}=\hbar_{\gamma} B_{\sigma}^{2} /\left(M c^{2}\right)$ (maximum laser cooling) and $\left.\angle E^{2}\right\rangle_{M}=2 k_{B} T B_{0}^{2} /\left(M c^{2}\right)$. In Table 1 are shown examples of the second order Doppler shift and $\left\langle E^{2}\right\rangle$ for single ions in $r f$ and Penning traps.

For clouds of identical ions, we first consider the electric fields due to collisions between ions. For the rf trap, we neglect the energy in the micromotion since the ions are driven in phase, therefore ion collisional effects in the $\mathrm{rf}$ and Penning traps are treated the same. $\left\langle E^{2}\right\rangle$ will of course depend on the cloud density and temperature but some idea of the magnitude can be given by calculating the electric field for one ion on another at the distance of closest approach. Assuming the maximum energy available for closest approach is given by $3 k_{B} T$, we have $E_{\max }=6.7 \times 10^{-8} \mathrm{~V} / \mathrm{cm}(\gamma / 2 \pi=10 \mathrm{MHz}$ and $\max -$

TABLE 1. Fractional second order Doppler shifts, Stark fields, and classical rms axial amplitudes $\left(z_{\text {rns }}\right)$ for single ions in $r f$ and Penning traps. When $\gamma / 2 \pi$ is given, we assume maximum theoretical laser cooling $\left(\Omega_{i} \ll \gamma\right)$. For both traps we assume $M=100 u$. For the $\operatorname{rf}$ trap $\Omega / 2 \pi\left(r f\right.$ drive frequency) $=1 \mathrm{MHz}, A_{0}=$ $300 \mathrm{~V} / \mathrm{cm}^{2}$. For the Penning trap, $\omega_{z} / 2 \pi=20 \mathrm{kHz}, B=1 \mathrm{~T} . T$ is the temperature of the secular motion for the of trap and the temperature of the cyclotron and axial motion for the Penning trap. $\left\langle E^{2}\right\rangle_{z}$ is the mean square electric field for motion along the $z$ axis, $\left\langle E^{2}\right\rangle_{M}$ is the mean square "motional" electric field for the $\mathbf{v} \times \mathbf{B} / c$ force. Note that $z_{m i s}$ for the Penning trap can be reduced at the expense of increasing $<E^{2}>$.

\begin{tabular}{|c|c|c|c|c|c|}
\hline & $\gamma / 2 \pi$ & - & - & $10 \mathrm{MHz}$ & $1 \mathrm{kHz}$ \\
\hline \multirow{5}{*}{$\mathrm{rf}$} & $T(\mathrm{~K})$ & 300 & 4 & $2.4 \times 10^{-4}$ & $2.4 \times 10^{-8}$ \\
\hline & $\Delta \nu_{D} / \nu_{0}$ & $8.3 \times 10^{-13}$ & $1.1 \times 10^{-14}$ & $6.6 \times 10^{-19}$ & $6.6 \times 10^{-23}$ \\
\hline & $\left\langle E^{2}\right\rangle_{z}\left(V^{2} / \mathrm{cm}^{2}\right)$ & 210 & 2.8 & $1.7 \times 10^{-4}$ & $1.7 \times 10^{-8}$ \\
\hline & $\bar{z}_{\mathrm{rms}}(\mu \mathrm{m})$ & 170 & 20 & 0.15 & $1.5 \times 10^{-3}$ \\
\hline & $\Delta v_{D} / \nu_{0}$ & $4.2 \times 10^{-13}$ & $5.5 \times 10^{-15}$ & $3.3 \times 10^{-19}$ & $3.3 \times 10^{-23}$ \\
\hline \multirow{3}{*}{ Pen. } & $\begin{array}{l}<E^{2}>_{2} \\
\left(V^{2} / \mathrm{cm}^{2}\right)\end{array}$ & $4.2 \times 10^{-2}$ & $5.7 \times 10^{-4}$ & $3.4 \times 10^{-8}$ & $3.4 \times 10^{-12}$ \\
\hline & $\begin{array}{l}<E^{2}>_{M} \\
\left(V^{2} / \mathrm{cm}^{2}\right)\end{array}$ & 4.99 & 0.067 & $4.0 \times 10^{-6}$ & $4.0 \times 10^{-10}$ \\
\hline & $\bar{z}_{\mathrm{rms}}(\mu \mathrm{m})$ & 1300 & 145 & 1.1 & 0.011 \\
\hline
\end{tabular}


imum laser cooling) and $E_{\max }=7.4 \mathrm{~V} / \mathrm{cm}$ at $T=4 \mathrm{~K}$. Therefore at modest temperatures, ion-ion collision induced Stark shifts can be quite small.

For clouds of ions, other effects can contribute to Stark and second order Doppler shifts. We will consider only theoretical limits and therefore neglect effects such as $\mathrm{rf}$ heating which may be the real limitation in a practical experiment. We will assume that the secular motion in an rf trap and the axial and cyclotron modes in a Penning trap have been cooled to negligible values. For both traps we will assune that it is desirable to maximize the number of ions $N$.

Then in an rf trap we must consider the effects of the micromotion and corresponding electric fields for ions on the edge of the cloud. We impose the constraint that the maximum fractional second order Doppler shift not exceed a certain value $(\epsilon)$. Therefore for the $\mathrm{rf}$ spherical well we set $\Psi(r, z)=-\phi_{i}$, and with the condition on $\epsilon$ (the maximum second order Doppler effect is due to ions at $z=r_{i}, r=0$ ) we find

$$
N_{\max }=6.48 \times 10^{15} r_{i} M \epsilon
$$

when $M$ is in $\mathrm{u}$, and $r_{i}$ is the cloud radius.

For the Penning trap, the maximum second order Doppler effect is due to the magnetron motion of ions on the edge of the cloud $\left(r_{m}=r_{i}, z=0\right)$. We set $\phi_{i}(z)=$ $-\phi_{T}(z)$ and for a spherical cloud we find from Eqs. $(5-7)$,

$$
N_{\max }=1.96 \times 10^{13} B \sqrt{\epsilon}\left[r_{i}^{2}-\frac{440 M \sqrt{\epsilon}}{B} r_{i}\right]
$$

where $B$ is in tesla, $M$ in u. Negative solutions are unphysical because they correspond to parameters where the magnetron second order Doppler shift cannot be made as large as $\epsilon$ (for a spherical cloud).
For very small values of $\epsilon$, very low density ion clouds are required which implies very small applied potentials. From the condition $\phi_{i}=-\Psi_{T}$ for the rf trap we have (for the spherical well):

$$
\frac{2 e A \sigma^{2}}{M \Omega^{2}}=7.2 \times 10^{-8} \frac{N_{\max }}{r_{i}{ }^{3}}\left\lceil\frac{\mathrm{V}}{\mathrm{cm}^{2}}\right]
$$

and similarly, for the Penning trap we have:

$$
A=3.6 \times 10^{-8} \frac{N_{\max }}{r_{i}^{3}}\left(\frac{\mathrm{V}}{\mathrm{cm}^{2}}\right) \text {. }
$$

We can also calculate the corresponding electric fields. As before, for the rf trap, the maximum fields occur on ions for $z=r_{i}$ and $r=0$ and we have

$$
\left\langle E^{2}>_{z(\max )}=8 r_{i}^{2} A_{\sigma}^{2}=\frac{2 M \Omega^{2} N_{\max }}{r_{i}} .\right.
$$

In the Penning trap, the electric fields cancel along the $z$ axis. Along the radial direction,

$$
\left\langle E^{2}\right\rangle_{r(\max )}=\left(2 M c^{2} \epsilon /\left(e r_{i}\right)\right)^{2} \text {. }
$$

In Table 2 are shown some representative values of maximum numbers of stored ions, trap potentials, and Stark shifts for various values of $\epsilon$ and $r_{i}$ on clouds of ions. In certam configurations, second order Doppler and Stark shifts could still be a problem; however, with small enough numbers of ions these can be overcome. We note that in many cases, the electric fields from black-body radiation $\left(\left\langle E^{2}\right\rangle_{b b} \simeq(10 \mathrm{~V} / \mathrm{cm})^{2}\right)$ can be much larger than those due to trapping conditions $[78,89,90]$. Therefore, operation at reduced environmental temperatures may ultimately be required.

TABLE 2. Maximum numbers, pseudopotential well depths for $r f$ traps, applied potentials for Penning traps, and electric fields for "cold" spherical ion clouds in rf and Penning traps. A maximum fractional second order Doppler shift $\epsilon$ is assumed. The secular motion for the of trap and the axial and cyclotron motion for the Penning trap are assumed to be frozen out (i.e., cooled to negligible values). $r_{i}=$ ion cloud radius: $M=100 u, \Omega 2 \pi=1 \mathrm{MHz}, B$ $=1 T$.

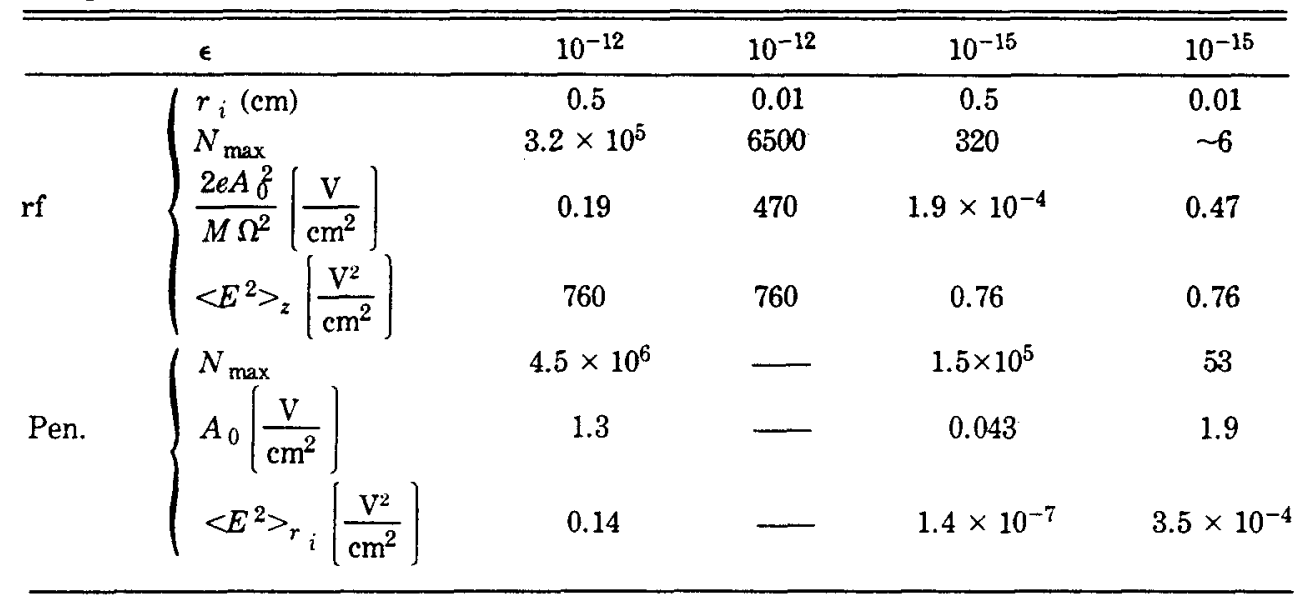




\section{Other Possibilities}

With the special features of the ion traps in mind, it is worthwhile speculating on possible future experiments. A very interesting class of experiments has already been initiated on multiply charged ions. Electron capture rates of $\mathrm{Ne}^{q+}(1 \leqslant q \leqslant 10)$ in an orbitron trap have recently been measured [14] and Penning traps are being developed for high $Z$ ion storage [91]. These experiments may pave the way for interesting spectroscopy on for example high $Z$ hydrogenic ions where precise theoretical calculations are available. Various energy separations become higher; for example the ${ }^{235} \mathrm{U}^{91+}$ ground state hfs transition may be in the visible [92]. In the future, more exotic species may be available for study such as antiprotons, $\mathrm{e}^{-} / \mathrm{e}^{-}$pairs in a Penning trap or $\mathrm{e}^{+} / \mathrm{e}^{-}, \mathrm{e}^{+} / \mathrm{p}^{-}$, $\mathrm{e}^{-/ \mathrm{p}^{+}}$or $\mathrm{p}^{+} / \mathrm{p}^{-}$pairs in an $\mathrm{rf}$ trap [92].

The Penning trap may be an ideal place to measure $g_{J}$ factors in certain atomic ions. First, because very small samples $(<100 \mu \mathrm{m})$ can be used, the effects of inhomogeneous fields are greatly reduced [81]. Second, atomic $g_{J}$ factors could be measured in terms of the Bohr magnetron by measuring the cyclotron frequency of free electrons in the same magnetic field. This technique has already been used to measure the $g_{J}$ factor in $S^{-}$[93]. More generally, these features plus the very high resolutions possible [82] should allow precise measurements of other magnetic-field-dependent structure. For example, deviations in the Breit-Rabi formula for hfs could be measured and it may be possible to measure the effects of nuclear diamagnetism [94].

From Tables 1 and 2, it is evident that Stark shifts can be very small on cooled ions; this suggests that the spectroscopy of Rydberg ions with minimal Stark shifts may be possible. With this in mind, perhaps a measurement of the Rydberg along the lines of Kleppner's proposal [95] is possible in an ion.

Finally, ion storage techniques might also be used for measurement devices, for example, to measure magnetic fields [96] or frequencies of lasers [45, 97].

Quite generally, it is now feasible to think of experiments on stored ions where the perturbations are extremely small. Hopefully, this can lead to many new precision experiments other than the ones discussed here.

The preparation of this paper was supported in part by the Air Force Office of Scientific Research and the Office of Naval Research. The author thanks W. M. Itano, G. Dunn, J. C. Bergquist, R. E. Drullinger and F. L. Walls for a critical reading of the manuscript and $R$. Ray and B. Barrett for manuscript typing.

\section{References}

[1] H. G. Dehmelt, Adv. At. Mol. Phys. 3, 53 (1967); 5, 109 (1969).

[2] F. G. Major and J. P. Schermann, Bull. Am. Phys. Soc. 16, 838 (1971).

[3] C. Audoin private communication (1979).

[4] F. G. Major, J. de Phys. (Paris) 38, L-221 (1977).

[5] D. Wineland, P. Ekstrom, and H. Dehmelt, Phys. Rev. Lett. 31,1279 (1973)

[6] R. S. Van Dyck, Jr., P. B. Schwinberg, and H. G. Dehmelt, in New Frontiers in High-Energy Physics, Ed. by B. M. Kursunoglu, A. Perlmutter, and L. F. Scott (Plenum, New York, 1978), p. 159; H. G. Dehmelt, in Atomic Physics 7, Ed. by D. Kleppner and P. M. Pipkin (Plenum, New York, 1981), p. 337.
[7] W. Neuhauser, M. Hohenstatt, P. E. Toschek, and H. Dehmelt, Phys. Rev. A 22, 1137 (1980).

[8] D. J. Wineland and W. M. Itano, Phys. Lett. 82A, 75 (1981).

[9] W. A. M. Blumberg, R. M. Jopson, and D. J. Larson, Phys. Rev. Lett. 40, 1320 (1978).

[10] W. A. M. Blumberg, W. M. Itano, and D. J. Larson, Phys. Rev. A 19, 139 (1979).

[11] R. T. McIver, Jr. in Lecture Notes in Chemistry, Vol. 7, Ed. by H. Hartmann and K. P. Wanczek (Springer-Verlag, New York, 1978), p. 97.

[12] F. L. Walls and G. H. Dunn, Phys. Today 27(8), 30 (August, 1974).

[13] H. A. Schuessler, Metrologia 13, 109 (1977); D. A. Church and H. M. Holzscheiter, Chem. Phys. Lett. 76, 109 (1980).

[14] C. R. Vane, M. H. Prior, and Richard Marrus, Phys. Rev. Lett. 46, 107 (1981).

[15] J. H. Malmberg and C. F. Driscoll, Phys. Rev. Lett. 44, 654 (1980); T. M. O'Neil, Proc. Inter. Conf. on Plasma Physics, II (April 1980).

[16] H. A. Schuessler, in Progress in Atomic Spectroscopy Part $B$, Ed. by W. Hanle and $\mathrm{H}$. Kleinpoppen (Plenum, New York, 1979), p. 999.

[17] G. Werth, Acta Phys. Polon. A61, 213 (1982).

[18] W. Paul, O. Osberghaus, and E. Fisher, Forchungsber. Wirtsch. Verkehrsministeriums Nordhein-Westfalen No. 415 (1958).

[19] F. Plumelle, M. Desaintfuscien, J. L. Duchene, and C. Audoin, Optics Commun. 34, 71 (1980); F. Plumelle, Thesis, Universite de Paris-Sud, Orsay (1979).

[20] R. Blatt and G. Werth, Z. Phys. 299, 94 (1981). Also see these proceedings.

[21] W. Neuhauser, M. Hohenstatt, P. Toschek, and H. G. Dehmelt, Phys. Rev. Lett. 41, 233 (1978); W. Neuhauser, M. Hohenstatt, P. Toschek, and H. G. Dehmelt, Appl. Phys. 17, 123 (1978).

[22] For a review see A. Ashkin, Science 210, 1081 (1980).

[23] F. M. Penning, Physica 3, 873 (1936).

[24] J. Byrne and P. S. Farago, Proc. Phys. Soc. (London) 86, 801 (1965).

[25] D. J. Wineland, R. E. Drullinger, and F. L. Walls, Phys. Rev. Lett. 40, 1639 (1978); R. E. Drullinger, D. J. Wineland, and J. C. Bergquist, Appl. Phys. 22, 365 (1980).

[26] D. J. Wineland and H. G. Dehmelt, Int. J. Mass. Spectr. Ion Phys. 16, 338 (1975); and erratum, 19, 251 (1976); H. G. Dehmelt, Nature 262, 777 (1976).

[27] See for example: S. A. Prasad and T. M. O'Neil, Phys. Fluids 22, 278 (1979).

[28] K. H. Kingdon, Phys. Rev. 21, 408 (1923)

[29] M. H. Prior, Phys. Rev. Lett. 29, 611 (1972).

[30] M. H. Prior and E. C. Wang, Phys. Rev. Lett. 35, 29 (1975).

[31] R. D. Knight, Appl. Phys. Lett. 38, 221 (1981)

[32] The case of a purely logarithmic potential has been investigated by R. H. Hooverman, J. Appl. Phys. 34, 3505 (1963). See also R. R. Lewis, J. Appl. Phys. 53, 3975 (1982).

[33] C. E. Johnson, private communication.

[34] A. Rich and J. C. Wesley, Rev. Mod. Phys. 44, 250 (1972). Also see the review by $A$. Rich, these proceedings.

[35] G. Gräff, E. Klempt, and G. Werth, Z. Phys. 222, 201 (1969).

[36] G. Gräff and M. Holzscheiter, Phys. Lett. 79A, 380 (1980).

[37] D. A. Church and B. Mokri, Z. Phys. 244, 6 (1971).

[38] F. L. Walls and T. Stein, Phys. Rev. Lett 31, 975 (1973).

[39] S. Liebes, Jr., and P. A. Franken, Phys. Rev. 116, 633 (1959).

[40] J. C. Wesley and A. Rich, Phys. Rev. A 4, 1341 (1971).

[41] R. S. Van Dyck, Jr., P. B. Schwinberg, and H. G. Dehmelt, Phys. Rev. Lett. 38, 310 (1977).

[42] P. Ekstrom and D. Wineland, Scientific American 243, August (1980).

[43] R. S. Van Dyck, Jr., D. J. Wineland, P. A. Ekstrom, and H. G. Dehmelt, Appl. Phys. Lett. 28, 446 (1976).

[44] D. J. Wineland and W. M. Itano, Phys. Rev. A 20, 1521 (1979).

[45] D. J. Wineland, J. Appl. Phys. 50, 2528 (1979).

[46] R. S. Van Dyck, Jr., P. B. Schwinberg, and H. G. Dehmelt, Bull. Am. Phys. Soc. 24, 758 (1979). 
[47j r. B. Schwinberg, R. S. Van Dyck, Jr., and H. G. Dehmelt, Phys. Rev. Lett. 24, 1679 (1981). Also see these proceedings.

[48] G. Gabrielse and R. S. Van Dyck, Jr., Bull. Am. Phys. Soc. 26. 598 (1981).

[49] H. Dehmelt, R. Van Dyck, P. Schwinberg, and G. Gabrielse, Bull. Am. Phys. Soc. 24, 675 (1979); and H. G. Dehmelt and G. Gabrielse, Bull. Am. Phys. Soc. 24, 758 (1979).

[50] P. B. Schwinberg and R. S. Van Dyck, Jr., Bull. Am. Phys. Soc. 24, 1202 (1979); Bull. Am. Phys. Soc. 26, 598 (1981).

[51] G. Gabrielse and H. G. Dehmelt, Bull. Am. Phys. Soc. 25 , 1149 (1980). Also see these proceedings.

[52] P. B. Schwinberg and R. S. Van Dyck, Jr., Bull Am. Phys. Soc. 23, 40 (1978).

[53] R. S. Van Dyck, Jr., P. B. Schwinberg, and S. H. Bailey, in Atomic Masses and Fundamental Constants 6, Ed. by J. A. Nolen, Jr., and W. Benenson (Plenum, New York, 1980), p. 173; R. S. Van Dyck, Jr., and P. B. Schwinberg, these proceedings.

[54] G. Gärtner and E. Klempt, Z. Phys. A 287, 1 (1978).

[55] G. Gräff, H. Kalinowsky, and J. Traut, Z. Phys. 296, 35 (1980). Also see these proceedings.

[56] D. J. Wineland and H. G. Dehmelt, J. Appl. Phys. 46, 919 (1975).

[57] D. J. Wineland, Bull. Am. Phys. Soc. 18, 1573 (1973).

[58] A. M. Wapstra and K. Bos, Atomic Data and Nuc. Data Tables 19, 177 (1977).

[59] R. Hegstrom, private communication.

[60] M. H. Prior and H. A. Shugart, Phys. Rev. Lett. 27, 902 (1971).

[61] R. D. Knight and M. H. Prior, Phys. Rev. 21, 179 (1980).

[62] R. Schneider and G. Werth, Z. Phys. 293, 103 (1980).

[63] P. B. Schwinberg, R. S. Van Dyck, Jr., and H. G. Dehmelt, Phys. Lett. 81A, 119 (1981).

[64] S. C. Menasian and H. G. Dehmelt, Bull. Am. Phys. Soc. 18, 408 (1973); F. J. Grieman, B. H. Mahan, and A. O'Keefe, J. Chem. Phys. 72, 4246 (1980).

[65] H. A. Schuessler, E. N. Fortson, and H. G. Dehmelt, Phys. Rev. 187, 5 (1969).

[66] M. H. Prior and E. C. Wang, Phys. Rev, Lett. 35, 29 (1975); and Phys. Rev. A 16, 6 (1977).

[67] F. G. Major, NASA Report X-521.69.167, Goddard Space Flight Center (1969).

[68] H. A. Schuessler, Metrologia 7, 103 (1971).

[69] F. G. Major and G. Werth, Phys. Rev. Lett. 30, 1155 (1973); F. G. Major and G. Werth, Appl. Phys. 15, 201 (1978)

[70] H. Dehmelt, Bull. Am. Phys. Soc. 18, 1521 (1973); H. Dehmelt, Bull. Am. Phys. Soc. 20, 60 (1975); D. J. Wineland and H. Dehmelt, Bull. Am. Phys. Soc. 20, 637 (1975); H. Dehmelt, IEEE Trans. Instrum. Meas. IM-31, 83 (1982).

[71] P. L. Bender, J. L. Hall, R. H. Garstang, F. M. J. Pichanick, W. W. Smith, R. L. Barger, and J. B. West, Bull. Am. Phys. Soc. 21, 599 (1976).

[72] F. Strumia, Proc. 32nd Ann. Symp. Freq. Control, June 1978, p. 444. (Copies available from Electronic Industries Assn., 2001 Eye St., NW., Washington, DC 20006.)
[73] F. L. Walls, D. J. Wineland, and R. E. Drullinger, Proc. 32nd Ann. Symp. Freq. Control, June 1978, p. 453. (Copies available from Electronic Industries Assn., 2001 Eye St., NW. Washington. DC 20006.)

[74] M. D. McGuire, R. Petsch, and G. Werth, Phys. Rev. A 17 1999 (1978). M. D. McGuire, Bull. Am. Phys. Soc. 26, 615 (1981).

[75] W. M. Itano and D. J. Wineland, Bull. Am. Phys. Soc. 24, 1185 (1979).

[76] M. Jardino, M. Desaintfuscien, R. Barillet, J. Viennet, P. Petit, and C. Audoin, Appl. Phys. 24, 1 (1981).

[77] P. E. Toschek and W. Neuhauser, in Atomic Physics 7, Ed. by D. Kleppner and F. M. Pipkin (Plenum, New York, 1981), p. 529.

[78] D. J. Wineland, W. M. Itano, J. C. Bergquist, and F. L. Walls, Proc. 35th Ann. Symp. Freq. Control, June, 1981. (Copies available from Electronic Industries Assn., 2001 Eye St., NW., Washington, DC 20006.) Also see W. M. Itano, D. J. Wineland, J. C. Bergquist and F. L. Walls, these proceedings.

[79] T. W. Hänsch and A. L. Schawlow, Opt. Commun. 13, 68 (1975).

[80] M. H. Prior and R. D. Knight, Opt. Comm. 35, 54 (1980).

[81] D. J. Wineland, J. C. Bergquist, W. M. Itano, and R. E. Drullinger, Opt. Lett. 5, 245 (1980).

[82] W. M. Itano and D. J. Wineland, Phys. Rev. A 24, 1364 (1981).

[83] W. Nagourney and H. Dehmelt, Bull. Am. Phys. Soc. 26 (1981).

[84] J. L. Hall, Science 202, 147 (1978); in Atomic Physics 7, Ed. by D. Kleppner and F. M. Pipkin (Plenum, New York, 1981), p. 267.

[85] D. J. Wineland, Proc. 11th Ann. Precise Time and Time Interval Meeting (NASA Publ. 2129, Nov. 1979), p. 81.

[86] W. Neuhauser, M. Hohenstatt, P. Toschek, and H. Dehmelt, Proc. 5th Int. Conf. on Spectral Lineshapes, West Berlin, July 1980.

[87] W. M. Itano and D. J. Wineland, Phys. Rev. A 25, 35 (1982).

[88] R. D. Haun, Jr., and J. R. Zacharias, Phys. Rev. 107, 107 (1957).

[89] T. F. Gallagher and W. E. Cooke, Phys. Rev. Lett. 42, 835 (1979).

[90] W. M. Itano, L. L. Lewis, and D. J. Wineland, Phys. Rev. A 25, 1233 (1982).

[91] D. A. Church, in Physics of Electron and Atomic Collisions, Ed. by S. Datz (North Holland, 1982), p. 533.

[92] H. G. Dehmelt, R. S. Van Dyck, Jr., P. B. Schwinberg, and G. Gabrielse, Bull. Am. Phys. Soc. 24, 757 (1979).

[93] R. M. Jopson and D. J. Larson, Phys. Rev. Lett. 47, 789 (1981).

[94] N. P. Economou, S. J. Lipson, and D. J. Larson, Phys. Rev. Lett. 38, 1394 (1977)

[95] D. Kleppner, Bull. Am. Phys. Soc. 20, 1458 (1975).

[96] P. B. Schwinberg and R. S. Van Dyck, Bull. Am. Phys. Soc. 24, 35 (1979).

[97] J. C. Bergquist and D. J. Wineland, Proc. 33rd Ann. Symp. Freq. Control, June 1979. (Copies available from Electronic Industries Assn., 2001 Eve St., NW., Washington, DC 20006.) 